Laura Olinda Bregiero Fernandes Costa ${ }^{1}$

Aline de Oliveira Riberro Viana ${ }^{2}$

MÔNICA DE OLIVEIRA ${ }^{3}$

Artigos originais

Palavras-chaves

Síndrome do ovário policístico Resistência a insulina

Sindrome X metabólica

Obesidade

Hiperandrogenismo

Keywords

Polycystic ovary syndrome Insulin resistance

Metabolic syndrome X

Obesity

Hyperandrogenism

\title{
Prevalência da síndrome metabólica em portadoras da síndrome dos ovários policísticos
}

\author{
Prevalence of the metabolic syndrome in women \\ with polycystic ovary syndrome
}

Resumo

OBJETIVO: avaliar a prevalência da síndrome metabólica nas portadoras da síndrome dos ovários policísticos (SOP). MÉTODOS: foram avaliadas 46 mulheres com SOP, segundo os critérios de Rotterdam (2003), e 44 mulheres saudáveis, com ciclos menstruais regulares, ausência de hiperandrogenismo clínico ou laboratorial e de microcistos ovarianos ao ultra-som pélvico (grupo controle). Para o diagnóstico da síndrome metabólica foram considerados os critérios do National Cholesterol Education Program (NCEP, 2002) e da International Diabetes Federation (IDF, 2005). RESULTADOS: a prevalência da síndrome metabólica foi de 30,4\% nas portadoras da SOP e de 6,8\% no grupo controle, segundo critérios do NCEP, e de 32,6 e 9,1\% nas pacientes com SOP e controles, respectivamente, segundo os critérios da IDF. A diferença entre estas proporções foi estatisticamente significante $(p=0,004)$, independente do critério utilizado, do IMC e da idade. Ao analisarmos cada componente individual da síndrome metabólica, observamos que a anormalidade metabólica mais freqüente nas portadoras da SOP foi o HDL colesterol abaixo de $50 \mathrm{mg} / \mathrm{dL}$, ocorrendo em $52,2 \%$ dos casos. As prevalências da glicemia de jejum superior a $110 \mathrm{mg} / \mathrm{dL}(4,3 \%)$, circunferência abdominal $>88 \mathrm{~cm}(47,8 \%)$, e pressão arterial $>130 / 85$ mmHg $(28,2 \%)$ foram significativamente maior nas portadoras da SOP. CONCLUSÕES: a síndrome metabólica é significativamente mais freqüente nas pacientes com SOP que na população geral, independente da idade e do IMC, o que pode significar um maior risco cardiovascular nestas pacientes.

\section{Abstract}

PURPOSE: to evaluate the prevalence of metabolic syndrome in women with polycystic ovary syndrome (PCOS). METHODS: forly six women with PCOS, in accord with Rotterdam criteria (2003), and 44 women with regular menses, without any clinical or laboratorial hyperandrogenism features, and no ultrasonographic ovarian microcysts (control group) were evaluated. For metabolic syndrome, the National Cholesterol Education Program (NCEP, 2002) and the International Diabetes Federation (IDF, 2005) guidelines were considered. RESULTS: the prevalence of metabolic syndrome were $30.4 \%$ (NCEP) and $32.6 \%$ (IDF) for the women with PCOS, nearly 4-fold higher than that reported for the control group ( $p<0.004)$, which were $6.8 \%$ (NCEP) and $9.1 \%$ (IDF). Women with PCOS had persistently higher prevalence rates of the metabolic syndrome, regardless of matched age and body mass index. The most prevalent factor of the metabolic syndrome among the PCOS subjects was low serum HDL cholesterol which was below $50 \mathrm{mg} / \mathrm{dl}(52.2 \%)$. Waist circumference above $88 \mathrm{~cm}$ $(47.8 \%)$, blood pressure above $130 / 85 \mathrm{mmHg}$ and fasting glycemia above $110 \mathrm{mg} / \mathrm{dl}(4.3 \%)$ were significantly more frequent among women with PCOS than among control women. CONCLUSIONS: the metabolic syndrome is significantly more frequent in women with PCOS, placing them at higher risk for cardiovascular disease.

Correspondência:

Laura Olinda Bregieiro Fernandes Costo Rua Bruno Maia, 217, apto. 1001 - Gracas - CEP 52011-110 Recife/PE - Fone: (81) 3221-2017 - Fax: (81) 3241-5946 E-mail: heliolaura@yahoo.com.br

Recebido $27 / 10 / 2006$

Aceito com modificacocoes $11 / 12 / 2006$
Departamento Materno-Infantil da Faculdade de Ciências Médicas da Universidade de Pernambuco - UPE - Recife (PE), Brasil. ' Professora Adjunta da Disciplina de Tocoginecologia da Faculdade de Ciências Médicas da Universidade de Pernambuco - UPE - Recife (PE), Brasil.

${ }^{2}$ Acadêmica da Faculdade de Ciências Médicas da Universidade de Pernambuco - UPE - Recife (PE), Brasil.

${ }_{3}^{3}$ Pós-graduanda em Tocoginecologia da Faculdade de Ciências Médicas da Universidade de Pernambuco - UPE - Recife (PE), Brasil. 
A síndrome dos ovários policísticos (SOP) é uma doença complexa e heterogênea que afeta, aproximadamente, $5-10 \%$ das mulheres em idade reprodutiva, sendo a desordem endócrina mais comum em americanas pré-menopáusicas ${ }^{1}$. Caracteriza-se pela presença de anovulação, infertilidade e hiperandrogenismo e está, freqüentemente, associada à obesidade, dislipidemia, hipertensão e diabetes tipo $\mathrm{II}^{2}$.

Estudos genéticos sugerem que a SOP é uma desordem complexa e multigênica. Variantes genômicas em genes relacionados à biossíntese, regulação e ação dos andrógenos, dos receptores androgênicos, à ação e à secreção da insulina, à secreção e à ação das gonadotrofinas e à síntese e metabolismo do ácido retinóico, assim como genótipos pró-inflamatórios (variantes dos genes do TNF- $\alpha$, IL-6) podem estar envolvidos na predisposição genética da $\mathrm{SOP}^{2,3}$.

A resistência à insulina e a hiperinsulinemia compensatória parecem desempenhar um papel importante na etiopatogenia da SOP ${ }^{4}$. Em nível central, a insulina parece estar envolvida na secreção anormal do LH e, em nível periférico, promove a secreção ovariana de andrógenos, por meio do aumento da expressão do gene $\mathrm{C} \gamma \mathrm{P} 17$ e da atividade do citocromo P450c17, tendo ação sinérgica com o LH, tanto diretamente como pelo estímulo da secreção do fator de crescimento símile à insulina 1 (IGF-1). A insulina diminui a síntese hepática de IGFBP-1 e da globulina ligadora dos hormônios sexuais (SHBG), aumentando os níveis de andrógenos livres ${ }^{2}$. Recentemente, foi proposta a existência, na SOP, de uma hipersensibilidade das células da teca à ação da insulina, defeito este intrínseco e não secundário ao estímulo crônico pelo LH e que pode ocorrer na ausência de resistência à insulina global ou hiperinsulinemia ${ }^{5}$.

Parece manifestar-se de diferentes formas nas diversas fases da vida da mulher: na vida intra-útero, traduzida pelo baixo peso ao nascer, na peripuberdade, pela pubarca precoce, nas mulheres adultas, pelas desordens reprodutivas, acne, hirsutismo e, a médio e longo prazo, pelas desordens metabólicas e maior risco para o desenvolvimento da doença cardiovascular e de câncer ${ }^{2,6}$.

Além das anormalidades reprodutivas da anovulação crônica e do hiperandrogenismo, um percentual significativo das portadoras da SOP tem múltiplos fatores de risco cardiovascular associados à resistência insulínica, como a intolerância à glicose e diabetes tipo $2^{7-9}$. Há também várias evidências que sugerem um elevado risco para doença cardiovascular nas pacientes portadoras da $\mathrm{SOP}^{4,10-12}$. A resistência à insulina parece determinar um estado de ativação endotelial, caracte- rizado por inflamação subclínica que justificaria um maior risco cardiovascular ${ }^{4,12}$.

Muitas mulheres portadoras da SOP manifestam um fenótipo comum à síndrome metabólica, compartilhando características como obesidade, tolerância diminuída à glicose, hipertensão, doença macrovascular e dislipidemia $^{13}$. A síndrome metabólica tem sido considerada uma das mais importantes situações de risco para a doença cardiovascular, dobrando a taxa de mortalidade por coronariopatias ${ }^{14}$. Embora as mulheres obesas, principalmente aquelas com distribuição abdominal de gordura, tenham um risco evidente de desenvolver a síndrome metabólica, estudos têm mostrado que o risco aumenta significativamente em mulheres com sobrepeso ou mesmo com índice de massa corporal (IMC) dentro dos limites de normalidade, desde que a SOP esteja presente ${ }^{15}$.

Embora a fisiopatologia da síndrome metabólica não esteja claramente definida, evidências recentes têm se somado, no sentido de reforçar o papel da resistência insulínica como base da síndrome metabólica, assim como é descrito na fisiopatologia da SOP ${ }^{16}$. Demonstra-se, nas portadoras da síndrome metabólica, um aumento das citocinas pró-inflamatórias, tais como o fator de necrose tumoral alfa e a interleucina-6, produzidas pelo tecido adiposo em quantidades substanciais ${ }^{15}$, que parecem comprometer a ação da insulina na captação celular da glicose e induzir a reação inflamatória endotelial ${ }^{15}$.

A proteína C-reativa, um sensível marcador inflamatório de fase aguda, mostra uma correlação positiva com a resistência à insulina, hiperinsulinemia compensatória e algumas manifestações da síndrome metabólica, e uma associação independente com o risco de doenças coronarianas ${ }^{17}$. Assim, a resistência à insulina, associada à gordura visceral, parece resultar em disfunção endotelial, dano vascular, estresse oxidativo em nível endotelial e posterior formação da placa de ateroma ${ }^{18}$.

Atualmente, os critérios utilizados para o diagnóstico da síndrome metabólica representam um tema bastante polêmico. Um dos primeiros critérios diagnósticos a serem sugeridos pela Organização Mundial da Saúde (OMS) reforçava a intolerância à glicose ou diabete tipo II, conseqüentes à resistência a insulina, como componentes essenciais para o diagnóstico, acompanhadas de pelo menos dois dos seguintes critérios: hipertensão arterial, dislipidemia, obesidade ou microalbuminúria ${ }^{19}$. O Grupo Europeu de Estudos sobre Resistência à Insulina modificou os critérios da OMS, excluindo os portadores de diabetes, sendo necessárias, por outro lado, a comprovação da hiperinsulinemia e a medida da circunferência abdominal, como avaliação da obesidade ${ }^{20}$. Mais recentemente, o 
Third Report of the National Cholesterol Education Program (NCEP ${ }^{21}$ sugere que a síndrome metabólica seja definida pela presença de pelo menos três das seguintes anormalidades: circunferência abdominal maior que $88 \mathrm{~cm}$, glicose de jejum em níveis iguais ou superiores a $110 \mathrm{mg} / \mathrm{dL}$, triglicerídeos em níveis iguais ou superiores a $150 \mathrm{mg} / \mathrm{dL}$, HDL colesterol em níveis iguais ou inferiores a $50 \mathrm{mg} / \mathrm{dL}$ e pressão arterial de no mínimo 130/85 $\mathrm{mmHg}$. O uso dos critérios diagnósticos do NCEP vem sendo fortemente questionado, em função da imprecisão de alguns critérios de definição da síndrome metabólica e pela falta de aplicabilidade dos critérios, em diferentes grupos étnicos, especialmente em relação às medidas da circunferência abdominal ${ }^{22}$. A recente definição da síndrome metabólica pela International Diabetes Federation (IDF) considera a obesidade central, avaliada pela circunferência abdominal, um componente essencial para o diagnóstico da síndrome metabólica, devido às fortes evidências de associação com a doença cardiovascular e com os outros componentes da síndrome metabólica ${ }^{23}$. De acordo com a IDF, o diagnóstico pode ser definido, na mulher, pela presença de circunferência abdominal maior que $80 \mathrm{~cm}$ e, pelo menos, mais dois dos seguintes critérios: triglicerídeos em níveis iguais ou superiores a $150 \mathrm{mg} / \mathrm{dL}$, HDL colesterol em níveis iguais ou inferiores a $50 \mathrm{mg} / \mathrm{dL}$, pressão arterial acima de 130/85 mmHg e glicemia em níveis iguais ou superiores a $100 \mathrm{mg} / \mathrm{dl}$. Apesar de valores específicos para os diferentes grupos étnicos terem sido incorporados, os critérios diagnósticos da IDF ainda não são unanimemente aceitos. Pela praticidade e simplicidade, a definição do NCEP é, ainda, amplamente aceita e recomendada pela I Diretriz Brasileira de Diagnóstico e Tratamento da Síndrome Metabólica - I-DBSM ${ }^{24}$.

Atualmente, existem poucos estudos que avaliam a prevalência da síndrome metabólica em portadoras da SOP, sendo a maioria deles realizados na população de mulheres norte-americanas, na qual a obesidade é significativamente mais prevalente que nas européias ou sul-americanas ${ }^{25}$. Além dos poucos estudos, o conhecimento da prevalência da síndrome metabólica pode ser útil na identificação de um subgrupo de pacientes portadoras da SOP, cujo risco de evento cardiovascular parece ser mais elevado, o que justificaria uma abordagem mais direcionada à proteção endotelial.

Assim, o objetivo do presente trabalho é avaliar, em nosso meio, a prevalência e a frequiência de cada um dos componentes da síndrome metabólica em pacientes portadoras da SOP, de acordo com os critérios do NCEP e da IDF.

\section{Métodos}

O estudo tipo corte transversal, de caráter analítico, foi desenvolvido no Ambulatório de Endocrinologia Tocoginecológica do Centro Integrado de Saúde Amaury de Medeiros (CISAM) da Universidade de Pernambuco (UPE), no período de março de 2005 a janeiro de 2006. Após esclarecimento e consentimento, foram selecionadas 46 pacientes, entre 15 e 38 anos, portadoras da SOP, segundo os critérios do último Consenso Internacional - Consenso de Rotterdam ${ }^{26}$, que define a SOP pela presença de pelo menos dois dos seguintes critérios diagnósticos: 1 Irregularidade menstrual, definida como oligomenorréia (menos de seis ciclos menstruais por ano) ou amenorréia (ausência de ciclo menstrual por três ou mais meses); 2 Evidência clínica e/ou bioquímica de hiperandrogenismo, como hirsutismo, acne, recesso temporal ou pele oleosa ou testosterona total elevada (acima do limite superior de normalidade do método - superior a $0,82 \mathrm{ng} / \mathrm{mL}) ; 3$ Ultra-sonografia pélvica evidenciando 12 ou mais folículos em cada ovário medindo 2-9 $\mathrm{mm}$ de diâmetro e/ou volume ovariano superior a $10 \mathrm{~cm}^{3}$. Outras causas de hiperandrogenismo - hiperprolactinemia, hiperplasia congênita da supra-renal, disfunção tireoidiana, síndrome de Cushing e neoplasias secretantes de androgênios - eram excluídas.

O grupo controle foi constituído de mulheres, entre 15 e 38 anos, com ciclos menstruais regulares, ausência de sinais de hiperandrogenismo clínico ou laboratorial e de microcistos ovarianos à ultrasonografia pélvica, que procuravam o serviço para realizar o exame de citologia oncótica (prevenção do câncer do colo uterino) no CISAM e que aceitaram participar voluntariamente do estudo, após os devidos esclarecimentos.

Foram excluídas as pacientes grávidas, portadoras de diabetes mellitus, usuárias de medicação anticoncepcional ou hormonal nos últimos três meses anteriores à inclusão no estudo, em uso de corticoesteróide e estatinas nos últimos três meses ou em uso de medicação que altere a resistência insulínica (glitazonas e metformina) nos últimos três meses.

De acordo com a rotina do nosso serviço, todas as pacientes do estudo foram submetidas a um exame físico geral, para a avaliação da distribuição e quantidade de pêlos, presença de acantose nigricans e acne, cálculo do IMC e aferição da pressão arterial, a um exame ginecológico detalhado, a exames laboratoriais para dosagens hormonais e bioquímicas e à ultra-sonografia pélvica. Para a avaliação da 
distribuição e quantidade dos pêlos foi utilizado o escore de Ferriman-Gallwey modificado, sendo consideradas hirsutas as mulheres com escore superior a sete. Para o cálculo do IMC foi utilizada a fórmula IMC $=$ peso $(\mathrm{kg}) /$ altura $(\mathrm{m})^{2}$. As pressões arteriais diastólica e sistólica foram aferidas após pelo menos 60 minutos de repouso.

As pacientes com SOP foram submetidas a ultrasonografia pélvica e amostras de sangue foram colhidas de veia periférica de membro superior, observado jejum de 12 horas, em período matutino para dosagens hormonais e bioquímicas, durante o período de oligo/amenorréia, sem induzir artificialmente o fluxo menstrual. No caso de a menstruação ocorrer espontaneamente, antes da coleta do sangue ou da realização da ultra-sonografia pélvica, estas pacientes com SOP, assim como as do grupo controle, foram orientadas a comparecer para submeterem-se aos exames após o término da menstruação, na fase folicular, entre os dias cinco e sete do ciclo menstrual. Em cada amostra de sangue foram dosados os seguintes exames nas portadoras de SOP e controles: glicemia basal (70$100 \mathrm{mg} / \mathrm{dL}$, Automação Cobas Mira Plus - Roche), colesterol total (200-240 mg/dL, Automação Cobas Mira Plus - Roche), HDL colesterol (superior a 45 $\mathrm{mg} / \mathrm{dL}$, Automação Cobas Mira Plus - Roche), triglicérides (até $150 \mathrm{mg} / \mathrm{dL}$, Automação Cobas Mira Plus - Roche), LDL-colesterol (até $130 \mathrm{mg} / \mathrm{dL}$, obtido pela fórmula de Friedewald), TSH - hormônio estimulador da tiróide ultra-sensível (0,4-5,0 mUI/mL, E-ICMA ensaio multisítio - anticorpo monoclonal), prolactina (2,5-14,6 ng/mL, E-ICMA ensaio multisítio - anticorpo monoclonal), testosterona total (até $0,82 \mathrm{ng} / \mathrm{mL}$, E-ICMA ensaio multisítio - anticorpo monoclonal), LH - hormônio luteinizante $(5-20 \mathrm{mUI} / \mathrm{mL}$, EICMA ensaio multisítio - anticorpo monoclonal) e 17-OHP - 17-hidroxiprogesterona (até $300 \mathrm{ng} / \mathrm{mL}$, - radioimunoensaio). Os exames ultra-sonográficos pélvico/endovaginal foram realizados utilizando-se um aparelho Shimadsu 2200, sempre que possível pelo mesmo examinador.

A síndrome metabólica foi definida segundo os critérios do $\mathrm{NCEP}^{21}$, ou seja, pela presença de pelo menos três das seguintes anormalidades: circunferência abdominal maior que $88 \mathrm{~cm}$, glicose de jejum em níveis iguais ou superiores a $110 \mathrm{mg} / \mathrm{dL}$, triglicerídeos em níveis iguais ou superiores a $150 \mathrm{mg} / \mathrm{dL}$, HDL colesterol em níveis iguais ou inferiores a $50 \mathrm{mg} / \mathrm{dL}$ e pressão arterial de no mínimo $130 / 85 \mathrm{mmHg}$; e da IDF, segundo a qual o diagnóstico pode ser definido, na mulher, pela presença de circunferência abdominal maior que $80 \mathrm{~cm}$ e, pelo menos, mais dois dos seguin- tes critérios: triglicerídeos em níveis iguais ou superiores a $150 \mathrm{mg} / \mathrm{dL}$, HDL colesterol em níveis iguais ou inferiores a $50 \mathrm{mg} / \mathrm{dL}$, pressão arterial acima de $130 / 85 \mathrm{mmHg}$ e glicemia em níveis iguais ou superiores a $100 \mathrm{mg} / \mathrm{dL}^{23}$.

O cálculo do tamanho amostral foi feito para estudo de corte transversal no STATCALC versão seis, do Epi-Info 3.3 para Windows, tendo como base uma prevalência de síndrome metabólica de 36,80\% para os casos e 9,60\% para os controles, seguindo dados de Ehrmann et al. ${ }^{13}$. Foi utilizado um erro $\alpha$ de $5 \%$ com uma força estatística de $80 \%$, sendo a proporção de casos e controles de 1:1. Segundo estes critérios, o tamanho amostral para a realização deste estudo foi de 44 participantes em cada grupo.

Os dados foram digitados e armazenados em banco de dados do Excel. A análise estatística foi realizada no Epi-Info, versão 3.3. Para a comparação de variáveis contínuas, foi utilizada a análise de variância para o ajuste das diferenças do IMC e idade entre os grupos controle e com SOP. Para a avaliação de associação de variáveis, foi utilizado o teste do $\chi^{2}$. Considerou-se diferença estatisticamente significante se $\mathrm{p}<0,05$.

Todas as pacientes assinaram o termo de consentimento, após os devidos esclarecimentos, e o projeto foi aprovado pelo Comitê de Ética e Pesquisa do CISAM.

\section{Resultados}

A Tabela 1 mostra que as pacientes com SOP eram significativamente mais jovens que as controles e apresentavam IMC, circunferência abdominal, escore de Ferriman-Gallwey, pressão arterial diastólica e a frequiência de acne e acantose nigricans significativamente superiores às pacientes controles. Em relação às dosagens laboratoriais, os níveis de LH, testosterona, colesterol total e LDL-colesterol eram significativamente superiores nas pacientes com SOP.

A prevalência da síndrome metabólica foi de 30,4\% nas pacientes com SOP e 6,8\% nas pacientes controles, segundo os critérios do NCEP, e de 32,6 e 9,1\% nas pacientes com SOP e controles, respectivamente, segundo os critérios da IDF (Tabela 2). A diferença entre estas proporções foi estatisticamente significante.

A Tabela 3 mostra a prevalência da síndrome metabólica, segundo os critérios do NCEP, nas pacientes com SOP e controles, estratificadas por idade. Observa-se que a prevalência da síndrome foi quase duas vezes maior na faixa dos 30 aos 38 anos nas pacientes com SOP, comparadas aos controles. Não foi observado nenhum caso da síndrome metabólica em pacientes controles com idade menor ou igual a 19 anos e entre 20 e 29 
anos e, nas pacientes com SOP, a prevalência foi de 2,2 e 17,4\% nestas faixas etárias, respectivamente.

Os grupos controle e as portadoras da SOP foram estratificados por idade e IMC. A prevalência da síndrome metabólica foi maior nas pacientes com SOP quando comparada aos controles, independente da faixa etária. Também mostrou uma tendência a ser progressivamente maior, de acordo com o aumento do IMC, tanto na faixa etária dos 20 aos 29, como dos 30 aos 38 anos (Tabela 4).

A freqüência das anormalidades da síndrome metabólica, segundo NCEP, é mostrada na Tabela 5. O componente mais freqüente em ambos os grupos foi o HDL colesterol em níveis inferiores a $50 \mathrm{mg} / \mathrm{dL}$, porém sem diferença estatisticamente significante. A distribuição de gordura corporal tipo abdominal (cintura $>88$ $\mathrm{cm}$ ), pressão arterial superior a $130 / 85 \mathrm{mmHg}$ e níveis de glicemia superiores a $110 \mathrm{mg} / \mathrm{dL}$ apareceram em cerca de 48, 28 e 4,3\%, respectivamente, nas pacientes com SOP, freqüências estas significativamente maiores, quando comparadas às do grupo controle.

Tabela 1 - Valores médios e desvio padrão dos parâmetros clínicos, hormonais e bioquímicos nas pacientes com SOP e controles.

\begin{tabular}{|c|c|c|c|}
\hline $\begin{array}{l}\text { Parâmetros clínicos e } \\
\text { laboratoriais }\end{array}$ & $\begin{array}{c}\text { SOP } \\
(n=46)\end{array}$ & $\begin{array}{l}\text { Controle } \\
(n=44)\end{array}$ & $\mathbf{p}$ \\
\hline Idade (anos) & $24,1 \pm 5,6$ & $30,9 \pm 6,5$ & $<0,001$ \\
\hline IMC ( $\left(\mathbf{k g} / \mathbf{m}^{2}\right)$ & $28,8 \pm 6,7$ & $25,9 \pm 5,5$ & 0,020 \\
\hline Circunferência abdominal (cm) & $88,6 \pm 14,6$ & $81,9 \pm 11,5$ & 0,010 \\
\hline Escore de Ferriman-Gallwey & $6,8 \pm 4,3$ & $2,5 \pm 1,7$ & $<0,001$ \\
\hline Acne (\%) & 50,0 & 11,4 & 0,003 \\
\hline Acantose nigricans (\%) & 41,3 & 6,8 & $<0,001$ \\
\hline Pressão arterial sistólica (mmHg) & $119 \pm 16,6$ & $114,0 \pm 9,9$ & 0,10 \\
\hline Pressão arterial diastólica $(\mathrm{mmHg})$ & $79,7 \pm 10,6$ & $74,0 \pm 8,9$ & 0,008 \\
\hline Testosterona (ng/dL) & $0,5 \pm 0,3$ & $0,2 \pm 0,2$ & $<0,001$ \\
\hline LH (mUl/mL) & $12,7 \pm 8,6$ & $5,8 \pm 4,2$ & $<0,001$ \\
\hline Glicemia de jejum (mg/dL) & $87,3 \pm 10,2$ & $86 \pm 7,6$ & 0,500 \\
\hline Colesterol total (mg/dL) & $170,7 \pm 32,0$ & $151,4 \pm 37,0$ & 0,009 \\
\hline LDL (mg/dL) & $101,0 \pm 33,0$ & $79,3 \pm 29,8$ & 0,001 \\
\hline HDL (mg/dL) & $49,6 \pm 12,5$ & $53,6 \pm 11,1$ & 0,113 \\
\hline Triglicerídeos (mg/dL) & $97,1 \pm 51,5$ & $92,3 \pm 62,3$ & 0,680 \\
\hline TSH (UI/mL) & $1,8 \pm 0,7$ & $2,0 \pm 1,6$ & 0,690 \\
\hline Prolactina (ng/mL) & $18,6 \pm 8,5$ & $21,1 \pm 8,7$ & 0,180 \\
\hline 17 Hidroxiprogesterona (ng/mL) & $1,6 \pm 0,8$ & $1,54 \pm 0,8$ & 0,73 \\
\hline
\end{tabular}

\section{Discussão}

A resistência à insulina e a hiperinsulinemia compensatória desempenham um papel importante na etiopatogenia da $\mathrm{SOP}^{4,27}$ e da síndrome metabólica ${ }^{4}$, que apresentam características clínicas e laboratoriais comuns.

O objetivo do presente trabalho foi reforçar prévios relatos da literatura que mostraram uma prevalência mais elevada da síndrome metabólica em pacientes com SOP, comparadas à população geral. Os critérios

Tabela 2 - Prevalência da síndrome metabólica nas mulheres com síndrome dos ovários policísticos (SOP) e controles, segundo os critérios de NCEP e IDF.

\begin{tabular}{lcc} 
& \multicolumn{3}{c}{ Classificação } \\
Grupos & NCEP & IDF \\
& $n(\%)$ & $n(\%)$ \\
\hline SOP $(n=46)$ & $14(30,4)^{\star}$ & $15(32,6)^{\star \star}$ \\
Controles $(n=44)$ & $3(6,8)^{\star}$ & $4(9,1)^{\star \star}$ \\
\hline
\end{tabular}

${ }^{*} p<0,05 .{ }^{*} p<0,05$. NCEP $=$ Natinal Cholesterol Education Program; IDF=International Diabetes Federation.

Tabela 3 - Prevalência da síndrome metabólica nas mulheres com síndrome dos ovários policísticos (SOP) e controles, de acordo com a faixa etária.

\begin{tabular}{l|cccc}
\hline \multirow{2}{*}{ Grupos } & \multicolumn{4}{c}{ Faixa etária (anos) } \\
& $\leq 19$ & $20-29$ & $30-38$ & Total \\
\hline SOP $(n=46)$ & $1(2,2)$ & $8(17,4)$ & $5(10,8)$ & 14 \\
Controles $(n=44)$ & 0 & 0 & $3(6,8)$ & 3 \\
\hline
\end{tabular}

Tabela 4 - Prevalência da síndrome metabólica em mulheres (n/\%) com a síndrome de ovários policísticos (SOP) $(n=46)$ e controles $(n=44)$, estratificadas pela faixa etária e índice de massa corporal (IMC).

\begin{tabular}{|c|c|c|c|c|}
\hline \multirow{3}{*}{ Faixa efária } & \multicolumn{3}{|c|}{ IMC } & \multirow{3}{*}{ Total na faixa etária } \\
\hline & $<25$ & $25-30$ & $>30$ & \\
\hline & n (\%) & n (\%) & n (\%) & \\
\hline \multicolumn{5}{|l|}{ 20-29 anos } \\
\hline SOP (n=37) & 0 & $2(5,4)$ & $7(18,9)$ & $9(16,1)$ \\
\hline Controles ( $n=19)$ & 0 & 0 & 0 & 0 \\
\hline \multicolumn{5}{|l|}{ 30-38 anos } \\
\hline SOP $(n=9)$ & 0 & $1(11,1)$ & $4(44,5)$ & $5(14,7)$ \\
\hline Controles $(n=25)$ & 0 & $2(8,0)$ & $1(4,0)$ & $3(8,8)$ \\
\hline
\end{tabular}

Tabela 5 - Freqüência dos componentes da síndrome metabólica, nas mulheres com síndrome dos ovários policísticos (SOP) e grupo controle.

\begin{tabular}{|c|c|c|c|c|c|}
\hline \multirow{2}{*}{ Componente da síndrome metabólica } & \multicolumn{2}{|c|}{ SOP } & \multicolumn{2}{|c|}{ Controle } & \multirow{2}{*}{$\mathbf{p}$} \\
\hline & n & (\%) & n & (\%) & \\
\hline Glicemia de jejum >110 mg/dL & 02 & 4,3 & 0 & 0 & 0,02 \\
\hline HDL colesterol $<50 \mathrm{mg} / \mathrm{dL}$ & 24 & 52,2 & 18 & 40,9 & 0,28 \\
\hline Triglicerídeos >150 mg/dL & 04 & 8,7 & 05 & 11,4 & 0,67 \\
\hline Pressão arterial >130/85 mmHg & 13 & 28,3 & 03 & 6,8 & 0,007 \\
\hline Circunferência abdominal >88 cm & 22 & 47,8 & 13 & 29,5 & 0,07 \\
\hline
\end{tabular}


do NCEP e da IDF foram utilizados, neste estudo, para a avaliação da prevalência da síndrome metabólica, já que sua definição ainda é bastante discutida na literatura. Estudos recentes examinam a associação entre outros marcadores de risco e a doença cardiovascular, como a avaliação da gordura visceral por tomografia, dosagens da apoproteína $\mathrm{B}$, marcadores inflamatórios, tais como a proteína $\mathrm{C}$-reativa, fator de necrose tumoral e interleucina-6, marcadores de risco trombogênico, como o inibidor do ativador do plasminogênio tipo I e fibrinogênio, e marcadores da disfunção endotelial ${ }^{4}$; após resultados conclusivos, poderão ser incorporados aos critérios diagnósticos da síndrome metabólica.

Pudemos observar, em nosso estudo, que a prevalência da síndrome metabólica foi mais de quatro vezes superior nas portadoras da SOP comparadas às do grupo controle, tanto pelos critérios do NCEP como da IDF. Apridonidze et al. ${ }^{28}$ mostraram uma prevalência da síndrome duas vezes maior nas portadoras da SOP, segundo os critérios do NCEP, comparadas a mulheres, estratificadas por idade, que participaram de um grande estudo populacional americano, o Third National Health and Nutrition Examination Survey (NHANES III ${ }^{29}$. Outros estudos demonstraram prevalências da síndrome metabólica, em portadoras de SOP, que variaram de 33,4 a $47 \%$ também segundo os critérios do NCEP ${ }^{13,30,31}$. Em um recente estudo caso-controle, foi encontrada uma prevalência da síndrome metabólica de $11 \%$ em mulheres jovens com SOP. Entretanto, cerca de $86 \%$ delas apresentavam dislipidemia e espessamento da íntima-média da carótida comum ${ }^{16}$, mostrando que a lesão endotelial pode estar presente desde os primeiros anos da adolescência. Estes e outros autores reforçam a necessidade de rastrear precocemente a síndrome metabólica, desde a adolescência, em portadoras da $\mathrm{SOP}^{14,30}$. Outros autores, entretanto, não encontraram uma prevalência superior nas portadoras da SOP, comparada à da população geral, a despeito do fato de as pacientes com SOP terem apresentado a média do IMC, da circunferência abdominal, das pressões sistólica e diastólica e dos níveis de HDL colesterol superiores aos do grupo controle ${ }^{25}$.

Nas pacientes com SOP, a síndrome metabólica é diagnosticada usualmente antes do final da terceira década de vida (23\% das pacientes com menos de 19 anos, $45 \%$ daquelas entre 20 e 29 anos e $53 \%$ daquelas entre 30 e 39 anos, versus 0,6 e $15 \%$ das mulheres controles, respectivamente), com prevalência que se aproxima da observada em mulheres sem SOP entre 50 e 60 anos de idade ${ }^{13,28}$. Em nosso estudo, o grupo controle não foi pareado por idade, o que, sem dúvida, representa uma limitação metodológica, já que estudos mostram uma tendência ao aumento da prevalência da síndrome metabólica com o avançar da idade ${ }^{28}$. Quando estratificamos as pacientes por idade observamos que, tanto nas pacientes com SOP como nas controles, a síndrome metabólica foi mais prevalente em mulheres mais velhas, acima de 25 anos (apenas uma paciente do grupo com SOP tinha 21 anos). Porém, em todas as faixas etárias, a prevalência da síndrome metabólica foi maior nas portadoras da $\mathrm{SOP}$, quando comparadas às controles. Além disso, a média de idade das mulheres com SOP (24,1 anos) foi significativamente menor que nas pacientes controles (30,9 anos). Estes resultados atenuam a limitação metodológica do não pareamento do grupo controle por idade, já que nas pacientes com SOP, mesmo mais jovens que as controles, a prevalência da síndrome metabólica foi maior neste grupo. Se tivéssemos observado o contrário, ou seja, uma prevalência superior no grupo controle, certamente a idade atuaria como um fator de confusão, comprometendo a análise dos resultados. Notando que as pacientes com SOP eram significativamente mais jovens que as controles, podemos considerar nossos resultados preocupantes. Tendo em vista o fato de que a síndrome metabólica parece estar associada à resistência insulínica e conseqüentemente à doença cardiovascular e diabetes, este achado em mulheres jovens com SOP sugere que as mesmas apresentam um meio metabólico adverso que as expõe a maior risco para a ocorrência de tais eventos. Neste sentido, a Associação Americana de Endocrinologistas Clínicos (AACE) considera a presença da SOP como um dos critérios para a definição da síndrome metabólica ${ }^{32}$.

Um achado interessante no nosso estudo, observado também por Ehrmann et al. ${ }^{13}$, é que todas as portadoras da SOP que apresentavam também o diagnóstico da síndrome metabólica tinham IMC maior ou igual a $25 \mathrm{~kg} / \mathrm{m}^{2}$. Esta observação sugere uma relação entre síndrome metabólica, resistência insulínica e obesidade. A obesidade, considerada como importante determinante da hiperinsulinemia, parece ter um efeito independente, segundo alguns autores, no risco de desenvolver a síndrome metabólica ${ }^{13}$. Cerca de $50 \%$ por cento das mulheres com SOP são obesas e a maioria apresenta distribuição de gordura tipo visceral ou abdominal que, por sua vez, está associada a um estado de resistência à insulina, hiperinsulinemia compensatória e hiperandrogenismo ${ }^{33}$. Assim, a insulina, por meio de seus próprios receptores e dos receptores da IGF-1, tem ação sinérgica ao LH no estímulo da esteroidogênese nas células da teca, aumentando significativamente a produção ovariana de androgênios, em mulheres 
com SOP, tanto em mulheres obesas, como magras. A obesidade, principalmente a visceral, amplifica a resistência à insulina e a hiperinsulinemia e, portanto, a produção androgênica ovariana ${ }^{33}$. Um ciclo vicioso, assim, se instala nessas pacientes, no qual a resistência à insulina, a hiperinsulinemia compensatória e o excesso de androgênios se interrelacionam determinando uma situação de múltiplos riscos cardiovasculares, como a dislipidemia, a hipertensão arterial, intolerância à glicose e obesidade visceral, que compõem a síndrome metabólica ${ }^{34}$. Por outro lado, quando estratificamos por IMC e idade, a prevalência da síndrome metabólica mostra uma tendência a permanecer maior nas portadoras da SOP, tanto nas magras, como nas obesas ou com sobrepeso, apesar de o tamanho amostral nos referidos estratos terem sido pequenos para essa análise. Este dado sugere que a obesidade, por si, não explica as diferenças na prevalência da síndrome metabólica que podem estar relacionadas à idade, nas pacientes com SOP, o que também foi observado por outros autores ${ }^{13,28}$. É possível que a presença da SOP, independente da idade e do peso corporal, seja uma situação de risco elevado para o desenvolvimento da síndrome metabólica, possivelmente secundária a um mecanismo intrínseco de resistência à insulina ${ }^{27,35}$.

Das anormalidades presentes, segundo os critérios do NCEP ${ }^{21}$, os níveis de HDL colesterol foram os mais freqüentes, tanto no grupo com SOP como no grupo controle, sem diferença estatisticamente significante entre os grupos. Este achado é consistente com os relatos de outros autores que demonstraram uma prevalência de $66 \%$ de níveis de HDL colesterol abaixo de $50 \mathrm{mg} / \mathrm{dL}^{13}$. Recentes estudos têm mostrado que o HDL colesterol parece proteger o endotélio, por efeito direto na ativação da enzima óxido nítrico sintetase, responsável pela sín- tese de óxido nítrico, um vasodilatador arterial ${ }^{36}$. Baixos níveis séricos do HDL colesterol têm sido reconhecidos como um fator de risco cardiovascular, independente dos níveis de LDL-colesterol.

O segundo achado mais freqüente, também nos dois grupos, foi a circunferência abdominal superior a $88 \mathrm{~cm}$, porém, a freqüência foi significativamente maior no grupo com SOP. Um aumento na circunferência abdominal é a tradução clínica da obesidade visceral, que apresenta uma forte correlação com a hiperinsulinemia e o hiperandrogenismo característicos da $\mathrm{SOP}^{13}$.

A seguir, apareceu a pressão arterial superior a $130 / 85 \mathrm{mmHg}$, significativamente mais freqüente no grupo com SOP, comparada aos controles. Além disso, pôde-se observar que a pressão arterial, particularmente a pressão diastólica, era significativamente mais elevada nas pacientes com SOP. Entre as várias moléculas derivadas do endotélio, relacionadas à reatividade vascular, a endotelina-1 vem sendo considerada um dos mais potentes vasoconstritores, e elevados níveis têm sido relatados em estados insulino-resistentes ${ }^{37}$ e mais recentemente associados à $\mathrm{SOP}^{17}$. Isso poderia ser uma das razões do aumento da pressão arterial nas pacientes com SOP.

Concluiu-se que a síndrome metabólica é significativamente mais prevalente, aparece precocemente e parece ser independente da idade e do IMC nas pacientes com SOP do que nas mulheres saudáveis, o que pode significar um maior risco cardiovascular a médio e longo prazo. Estudos prospectivos são necessários para se identificarem os fatores clínicos, hormonais e metabólicos preditivos para o aparecimento da síndrome metabólica, assim como para reforçá-la como importante fator de risco cardiovascular nas mulheres portadoras da SOP.

\section{Referências}

1. Azziz R, Woods KS, Reyna R, Key TJ, Knochenhaver ES, Yildiz $B O$. The prevalence and features of the polycystic ovary syndrome in an unselected population. J Clin Endocrinol Metab. 2004; 89(6):2745-9.

2. Ehrmann DA. Polycystic ovary syndrome. N Engl J Med. 2004; 352(12):1223-36

3. Qin K, Ehrmann DA, Cox N, Refetoff S, Rosenfield RL. Identification of a functional polymorphism of the human type 517 beta-hydroxysteroid dehydrogenase gene associated with polycystic ovary syndrome. J Clin Endocrinol Metab. 2006; 91 (1):270-6.

4. Cussons AJ, Stuckey, BG, Watts GF. Cardiovascular disease in the polycystic ovary syndrome: new insights and perspectives. Atherosclerosis. 2006; 185(2):227-39.
5. Baillargeon JP, Nestler JE. Polycystic ovary syndrome: a syndrome of ovarian hypersensitivity of insulin? J Clin Endocrinol Metab. 2006; $91(1): 22-4$.

6. Ozanne SE, Fernandez-Twinn F, Hales $C N$. Fetal growth and adult diseases. Semin Perinatol. 2004; 28(1):81-7.

7. Bhatia V. Insulin resistance in polycystic ovary disease. South Med J. 2005; 98(9):903-10.

8. Vrbikova J, Cibula D, Dvorakova K, Stanicka S, Sindelka G, Hill M, et al. Insulin Sensitivity in women with polycystic ovary syndrome. J Clin Endocrinol Metab. 2004; 89(6):2942-5.

9. Trolle B, Lauszus FF. Risk factors for glucose intolerance in Danish women with polycystic ovary syndrome. Acta Obstet Gynecol Scand. 2005; 84(12):1192-6. 
10. Wild S, Pierpoint T, McKeighe P, Jacobs H. Cardiovascular disease in women with polycystic ovary syndrome at long-term follow-up: a retrospective cohort study. Clin Endocrinol (Oxf). 2000; 52(5):595-600

11. Legro RS. Polycystic ovary syndrome and cardiovascular disease: a premature association? Endocr Rev. 2003; 24(3):302-12.

12. Meyer $C, M c G r a t h B P$, Teede HJ. Overweight women with polycystic ovary syndrome have evidence of subclinical cardiovascular disease. J Clin Endocrinol Metab. 2005; 90(10): 5711-6.

13. Ehrmann DA, Liljenquist DR, Kasza K, Azziz R, Legro RS, Ghazzi $M N$, et al. Prevalence and predictors of the metabolic syndrome in women with polycystic ovary syndrome. J Clin Endocrinol Metabol. 2006; 91 (1):48-53.

14. Miller EL, Mitchell A. Metabolic syndrome: screening, diagnosis, and management. J Midwifery Womens Health. 2006; $51(3)$ : 141-51.

15. Gogia A, Agarwal PK. Metabolic syndrome. Indian J Med Sci. 2006; 60(2):72-81.

16. Vural B, Caliskan E, Turkoz E, Kilic T, Demirci A. Evaluation of metabolic syndrome frequency and premature carotid atherosclerosis in young women with polycystic ovary syndrome. Hum Reprod. 2005; 20(9):2409-13

17. Diamanti-Kandarakis E, Alexandraki K, Piperi C, Protogerou A, Katsikis I, Paterakis T, et al. Inflammatory and endothelial markers in women with polycystic ovary syndrome. Eur J Clin Invest. 2006; 36(10):691-7.

18. Kougias $P$, Chai $H$, Lin $P H, Y a o Q$, Lumsden $A B$, Chen C. Effects of adipocyte-derived cytokines on endothelium functions: implication of vascular disease. J Surg Res. 2005; 126(1):121-9.

19. Alberti KG, Zimmet PZ. Definition, diagnosis and classification of diabetes mellitus and its complications. Part 1: diagnosis and classification of diabetes mellitus provisional report of a WHO consultation. Diabet Med. 1998; 15(7):539-53.

20. Balkau B, Charles MA. Comment on the provisional report from the WHO consultation. European Group for the Study of Insulin Resistance (EGIR). Diabet Med. 1999; 16(5):442-3.

21. National Cholesterol Education Program (NCEP) Expert Panel on Detection, Evaluation, and Treatment of High Blood Cholesterol in Adults (Adult Treatment Panel III). Third Report of the National Cholesterol Education Program (NCEP) Expert Panel on Detection, Evaluation, and Treatment of High Blood Cholesterol in Adults (Adult Treatment Panel III) final report. Circulation. 2002; 106(25): 3143-421.

22. Kahn R, Buse J, Ferrannini E, Stern M; American Diabetes Association; European Association for the Study of Diabetes. The metabolic syndrome: time for a critical appraisal: joint statement from the American Diabetes Association and the European Association for the Study of Diabetes. Diabetes Care. 2005; 28(9):2289-304. Review.

23. Alberti KG, Zimmet P, Shaw J; IDF Epidemiology Task Force Consensus Group. The metabolic syndrome-a new worldwide definition. Lancet. 2005; 366(9491):1059-62.
24. Sociedade Brasileira de Hipertensão; Sociedade Brasileira de Cardiologia; Sociedade Brasileira de Endocrinologia e Metabologia; Sociedade Brasileira de Diabetes; Associação Brasileira para Estudos da Obesidade. I diretriz brasileira de diagnóstico e tratamento da síndrome metabólica. Arq Bras Cardiol. 2005; 84 Supl 1:3-28.

25. Vrbíkova J, Vondra K, Cibula D, Dvorakova K, Stanicka S, Sramkova $D$, et al. Metabolic syndrome in young Czech women with polycystic ovary syndrome. Hum Reprod. 2005; 20(12):3328-32.

26. Rotterdam ESHRE/ASRM-Sponsored PCOS Consensus Workshop Group. Revised 2003 consensus on diagnostic criteria and longterm health risks related to polycystic ovary syndrome. Fertil Steril. 2004; 81 (1): 19-25.

27. Lord J, Thomas R, Fox B, Acharya U, Wilkin T. The central issue? Visceral fat mass is a good marker of insulin resistance and metabolic disturbance in women with polycystic ovary syndrome. BJOG. 2006; 113(10):1203-9.

28. Apridonidze T, Essah PA, lourno M, Nestler JE. Prevalence and characteristics of metabolic syndrome in women with polycystic ovary syndrome. J Clin Endocrinol Metab. 2005; 90(4):1929-35.

29. Ford ES, Giles WH, Dietz WH. Prevalence of the metabolic syndrome among US adults: findings from the third National Health and Nutrition Examination Survey. JAMA. 2002; 287(3):356-9.

30. Dokras A, Bochner M, Hollinrake E, Markham S, Vanvoorhis B, Jagasia DH. Screening women with polycystic ovary syndrome for metabolic syndrome. Obstet Gynecol. 2005; 106(1):131-7.

31. Glueck CJ, Papanna R, Wang P, Goldenberg N, Sieve-Smith L. Incidence and treatment of metabolic syndrome in newly referred women with confirmed polycystic ovarian syndrome. Metabolism. 2003; 52(7):908-15.

32. Grundy SM, Cleeman Jl, Daniels SR, Donato KA, Eckel RH, Franklin BA, Gordon DJ, Krauss RM, Savage PJ, Smith SC Jr, Spertus JA, Costa F; American Heart Association; National Heart, Lung, and Blood Institute. Diagnosis and management of the metabolic syndrome: an American Heart Association/National Heart, Lung, and Blood Institute Scientific Statement. Circulation. 2005; 112 (17):2735-52.

33. Pasquali R. Obesity, fat distribuition and infertility. Maturitas. 2006; 54(4):363-71.

34. Pasquali R. Obesity and androgens: facts and perspectives. Fertil Steril. 2006; 85(5): 1319-40.

35. Barber TM, McCarthy MI, Wasst JA, Franks $S$. Obesity and polycystic ovary syndrome. Clin Endocrinol. 2006; 65(2):137-45.

36. Nofer JR, Van der Giet M, Tolle M, Wolinska I, Von Wnuck Lipinski $\mathrm{K}$, Baba HA, et al. HDL induces NO- dependet vasorelaxation via the lysophospholipid receptor S1P3. J Clin Invest. 2004; $113(4): 569-81$.

37. Potenza MA, Marasciulo FL, Chieppa DM, Brigiani GS, Formoso $G$, Quon M, Montagnani M. Insulin resistance in spontaneously hypertensive rats is associated with endothelial dysfunction characterized by imbalance between NO and ET-1 production. Am J Physiol Heart Circ Physiol. 2005; 289(2):H813-22. 